\title{
Through-the-Wall Radar Imaging for Heterogeneous Walls using Compressive Sensing
}

\author{
Eva Lagunas*, Moeness G. Amin ${ }^{\dagger}$, Fauzia Ahmad ${ }^{\dagger}$ \\ *Interdisciplinary Centre for Security, Reliability and Trust (SnT), University of Luxembourg, L-2721, Luxembourg \\ Email: eva.lagunas@uni.lu \\ ${ }^{\dagger}$ Center for Advanced Communications, Villanova University, Villanova, PA 19085, USA \\ Email: \{moeness.amin,fauzia.ahmad\}@villanova.edu
}

\begin{abstract}
Front wall reflections are considered one of the main challenges in sensing through walls using radar. This is especially true under sparse time-space or frequency-space sampling of radar returns which may be required for fast and efficient data acquisition. Unlike homogeneous walls, heterogeneous walls have frequency and space varying characteristics which violate the smooth surface assumption and cause significant residuals under commonly used wall clutter mitigation techniques. In the proposed approach, the phase shift and the amplitude of the wall reflections are estimated from the compressive measurements using a Maximum Likelihood Estimation (MLE) procedure. The estimated parameters are used to model electromagnetic (EM) wall returns, which are subsequently subtracted from the total radar returns, rendering wall-reduced and wall-free signals. Simulation results are provided, demonstrating the effectiveness of the proposed technique and showing its superiority over existing methods.
\end{abstract}

\section{INTRODUCTION}

Through-the-wall radar imaging (TWRI) [1]-[4] is an emerging technology that uses electromagnetic (EM) waves to penetrate man-made building materials and image targets behind visually opaque structures. Imaging of building interiors has recently been a subject of interest in both civilian and military contexts [5], [6]. One of the main challenges in TWRI is the detection and localization of stationary targets inside enclosed structures. This is attributed to the fact that targets radar cross-section (RCS) is much weaker than front wall EM backscatterings. Therefore, stationary targets cannot be generally detected without an effective removal of front wall clutter [7], [8].

Reduction of radar measurements due to logistics in data collections or fast data acquisition requirements make the above challenge even more formidable. If the front wall causes insignificant clutter, previous work on Compressive Sensing (CS) and 11-norm reconstruction techniques [9], [10] have shown that sparsity of the indoor scene can be exploited in TWRI to provide high-resolution images while alleviating the demand on acquisition and processing time [11]-[14]. Wall mitigation techniques in conjunction with CS were first considered in [15]-[17]. Direct application of these methods to the reduced data volume was shown to provide comparable performance to their full data volume counterparts. In [15][17], wall clutter reduction typically assumes spatial invariance of the wall reflections along the array dimension. While this condition complies best for walls with flat surface and antenna arrays parallel to front wall, it is clearly no longer satisfied when dealing with walls with non-planar surfaces. The approach in [18] relaxes the spatial invariance of the front wall and confined it to neighboring antenna positions; however, it required accurate calibration and was originally introduced to work on the full data volume and did not account for reduced data measurements.

Inspired by recent works on Ground Penetrating Radar (GPR) [19], this paper proposes a simple and effective wall mitigation technique using compressive measurements that can be used for planar and non-planar walls. In the proposed approach, the phase shift and the amplitude of the wall reflections are estimated from reduced measurements using a Maximum Likelihood Estimation (MLE) [20] procedure. The estimated parameters are used to model EM wall returns, which are subtracted from the total radar returns, rendering wall-free signals. Numerical simulations are provided which validate the effectiveness of the proposed technique and prove its superiority over existing techniques.

The remainder of the paper is organized as follows. In Section II we introduce the TWR received signal model and its relation with the radar image and we provide the CS fundamental formulation in TWRI context. Section III reviews conventional wall clutter mitigation methods. Section IV describes the proposed wall mitigation technique. Supporting simulation results are presented in Section $\mathrm{V}$ and concluding remarks are provided in Section VI.

\section{Signal Model}

Assume a synthetic aperture radar (SAR) is used in which a single antenna at one location transmits and receives the radar signal, then moves to the next location, and repeats the same operation along the axis parallel to a homogenous wall. Consider the homogeneous wall located along the $x$-axis, and the region to be imaged located beyond the wall along the positive $z$-axis. Assume $N$ antenna locations, with the $n$-th transceiver, located at $\mathbf{x}_{n}=\left(x_{n},-z_{\text {off }}\right)$, where $z_{\text {off }}$ is the wall standoff distance. The scene is illuminated with a steppedfrequency signal of $M$ frequencies, which are equispaced over the desired bandwidth $\omega_{M-1}-\omega_{0}$,

$$
\omega_{m}=\omega_{0}+m \Delta_{\omega} \quad m=0, \ldots, M-1
$$

where $\omega_{0}$ is the lowest frequency in the desired frequency band and $\Delta_{\omega}$ is the frequency step size. The reflections by the wall and any targets in the scene are measured only at the same transceiver location. Assuming the scene contains $P$ point targets, the output of the $n$-th transceiver corresponding 
to the $m$-th frequency is given by,

$$
y(m, n)=y_{\mathrm{w}}(m, n)+\sum_{p=0}^{P-1} \sigma_{p} e^{-j \omega_{m} \tau_{p, n}}
$$

where $y_{\mathrm{w}}(m, n)$ denotes the wall EM backscatterings, $\sigma_{p}$ is the complex reflectivity of the $p$-th target and $\tau_{p, n}$ is the two-way traveling time between the $n$-th antenna and the target. For through-the-wall propagation, $\tau_{p, n}$ will comprise the components corresponding to traveling distances before, through, and after the wall [21]. A reference wall reflection model for an average standoff distance $d$ from the surface can be stated as,

$$
y_{\mathrm{w}, \mathrm{ref}}(m)=e^{-j \omega_{m} \tau_{\mathrm{w}}}
$$

where $\tau_{\mathrm{w}}=2 d / c$. However, the actual wall reflection $y_{\mathrm{w}}(m, n)$ from a rough wall will be a scaled and phase-shifted version of $y_{\mathrm{w}, \mathrm{ref}}(m)$ as,

$$
y_{\mathrm{w}}(m, n)=\alpha_{m} y_{\mathrm{w}, \mathrm{ref}}(m) e^{j \omega_{m} \Delta \tau_{n}}
$$

where $\alpha_{m}$ is the scale and $\Delta \tau_{n}$ denotes the time shift with respect to the average time delay $\tau_{\mathrm{w}}$ for the $n$-th antenna location. Note that wall ringing or reverberation is not considered in (4). Therefore, the indoor targets are obscured due to the wall reflections, which are relatively stronger than target reflections.

Assume that the region of interest is divided into a finite number of pixels $N_{x} \times N_{z}$ in crossrange and downrange. In CSbased TWRI, the scene itself is usually considered sparse. The sparsity-driven approach operates under the assumption of the point target model and applies the sparsity condition directly to the scene, arguing that the point targets occupy no more than $P\left(\ll N_{x} \times N_{z}\right)$ pixels. Let $r(k, l), k=0, \ldots, N_{x}-1$, $l=0, \ldots, N_{z}-1$ be a weighted indicator function, which takes the value $\sigma_{p}$ if the $p$-th point target exists at the $(k, l)$-th pixel; otherwise, it is zero. With the values $r(k, l)$ lexicographically ordered into a column vector $\mathbf{r}$ of length $N_{x} N_{z}$, the received signal corresponding to the $n$-th antenna can be expressed in matrix-vector form as,

$$
\mathbf{y}_{n}=\boldsymbol{\Psi}_{n} \mathbf{r}
$$

where $\boldsymbol{\Psi}_{n}$ is a matrix of dimensions $M \times N_{x} N_{z}$, and for homogeneous walls, its $m$-th row is given by,

$$
\left[\boldsymbol{\Psi}_{n}\right]_{m}=\left[\begin{array}{lll}
e^{-j w_{m} \tau_{n, 00}} & \cdots & e^{-j w_{m} \tau_{n, N_{x} N_{z}}}
\end{array}\right]
$$

with $\quad \mathbf{y}_{n}=\left[\begin{array}{llll}y(0, n) & y(1, n) & \cdots & y(M-1, n)\end{array}\right]^{T}$ and $y(m, n)$ given in (2). Considering the measurement vector corresponding to all $N$ antennas, defined as,

$$
\mathbf{y}=\left[\begin{array}{llll}
\mathbf{y}_{0}^{T} & \mathbf{y}_{1}^{T} & \cdots & \mathbf{y}_{N-1}^{T}
\end{array}\right]^{T}
$$

the relationship between $\mathbf{y}$ and $\mathbf{r}$ is given by,

$$
\mathbf{y}=\mathbf{\Psi r}
$$

where

$$
\boldsymbol{\Psi}=\left[\begin{array}{llll}
\boldsymbol{\Psi}_{0}^{T} & \boldsymbol{\Psi}_{1}^{T} & \cdots & \boldsymbol{\Psi}_{N-1}^{T}
\end{array}\right]^{T} .
$$

The expression in (8) involves the full set of measurements made at the $N$ array locations using all $M$ frequencies.

Consider $\breve{\mathbf{y}}$, which is a vector of length $Q_{\mathrm{f}} Q_{\mathrm{n}}(\ll M N)$ consisting of elements chosen from $\mathbf{y}$ as follows,

$$
\breve{\mathbf{y}}=\Phi \mathbf{y}=\Phi \Psi \mathbf{r}
$$

where $\boldsymbol{\Phi}$ is a $Q_{\mathrm{f}} Q_{\mathrm{n}} \times M N$ matrix of the form,

$$
\boldsymbol{\Phi}=\left(\boldsymbol{\vartheta} \otimes \mathbf{I}_{Q_{\mathrm{f}}}\right) \cdot \operatorname{diag}\left\{\boldsymbol{\varphi}^{(0)}, \ldots, \boldsymbol{\varphi}^{(N-1)}\right\}
$$

In (11), $\otimes$ denotes the Kronecker product, $\mathbf{I}_{Q_{\mathrm{f}}}$ is a $Q_{\mathrm{f}} \times Q_{\mathrm{f}}$ identity matrix, $\vartheta$ is a $Q_{\mathrm{n}} \times N$ measurement matrix constructed by either uniformly or randomly selecting $Q_{\mathrm{n}}$ rows of an $N \times N$ identity matrix, and $\varphi^{(n)}, n=0,1, \ldots, N-1$, is a $Q_{\mathrm{f}} \times M$ measurement matrix constructed by uniformly or randomly selecting $Q_{\mathrm{f}}$ rows of an $M \times M$ identity matrix. We note that $\vartheta$ determines the reduced antenna locations, whereas $\varphi^{(n)}$ determines the reduced set of frequencies corresponding to the $n$th antenna location. The number of measurements $Q_{\mathrm{f}} Q_{\mathrm{n}}$ required to achieve successful CS reconstruction highly depends on the coherence between $\Phi$ and $\boldsymbol{\Psi}$. For the problem at hand, $\boldsymbol{\Phi}$ is the canonical basis and $\boldsymbol{\Psi}$ is similar to the Fourier basis, which have been shown to exhibit maximal incoherence [10]. Given $\breve{\mathbf{y}}$, we can recover $\mathbf{r}$ by solving the following equation,

$$
\hat{\mathbf{r}}=\arg \min _{\mathbf{r}}\|\mathbf{r}\|_{l_{1}} \quad \text { subject to } \quad \breve{\mathbf{y}} \approx \boldsymbol{\Phi} \Psi \mathbf{r}
$$

where $\|\mathbf{r}\|_{l_{1}}$ is simply the sum of the absolute values of the elements in $\mathbf{r}$. Several methods are available in the literature to solve the optimization problem in (12). In this paper, we consider Matching Pursuit (MP) to solve (12). More specifically, we use the Orthogonal Matching Pursuit (OMP) [22], which is known to provide a fast and easy to implement solution.

\section{EXISTING WALL MITIGATION TECHNIQUES}

Previous works thus far assumed a planar front wall which causes specular reflections towards the SAR [23], [24]. Assuming $\alpha_{m}=1$ and $\Delta \tau_{n}=0$ in (4), we note that the two-way traveling time from the wall to each of the antenna elements does not vary with the antenna location. Furthermore, as the wall is homogeneous and assumed to be of extent that is much larger than the beamwidth of the antenna, the wall return in (2) assumes the same value across the array aperture. Unlike $\tau_{\mathrm{w}}$, the time delay $\tau_{p, n}$ in (2) is different for each antenna location, since the signal path from the antenna to the target is different from one antenna to the other. For the $m$-th frequency, the received signal is a function of $n$ via the variable $\tau_{p, n}$. Therefore, we can rewrite (2) as,

$$
y_{\omega_{m}}(n)=v_{\omega_{m}}+\sum_{p=0}^{P-1} u_{p, \omega_{m}}(n)
$$

where $v_{\omega_{m}}=y_{\mathrm{w}, \mathrm{ref}}(m)$ and $u_{p, \omega_{m}}(n)=\sigma_{p} e^{-j \omega_{m} \tau_{p, n}}$. Thus, separating wall reflections from target reflections amounts to basically separating constant from non-constant valued signals across antennas, which can be performed by applying a proper spatial filter [23] across the antenna array. In its simplest form, the spatial filter, which removes, or significantly attenuates, the zero spatial frequency component, can be implemented as the subtraction of the average of the radar return across the antennas. That is,

$$
\tilde{y}_{\omega_{m}}(n)=y_{\omega_{m}}(n)-\bar{y}_{\omega_{m}}
$$

where

$$
\bar{y}_{\omega_{m}}=\frac{1}{N} \sum_{n=0}^{N-1} y_{\omega_{m}}(n)
$$




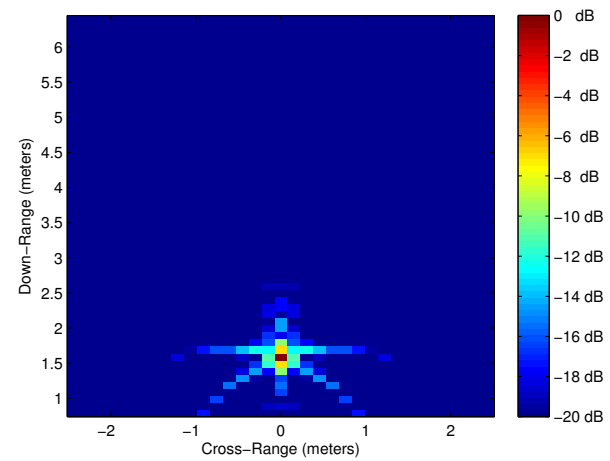

(a)

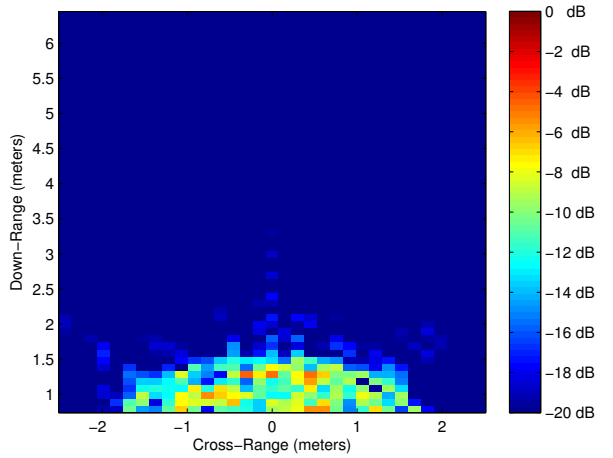

(b)

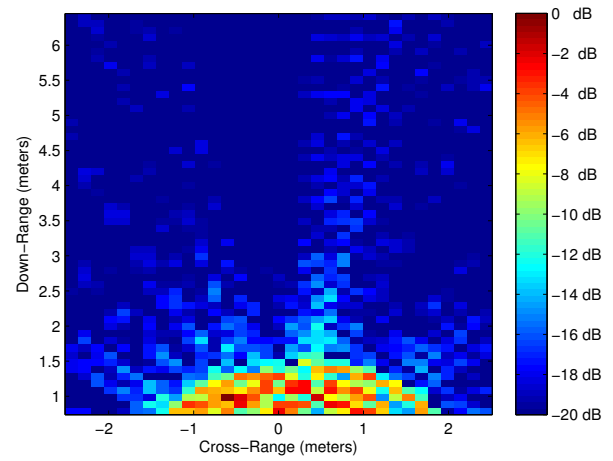

(c)

Fig. 1: Backprojected image: (a) Scene image with full data set when wall contribution is perfectly removed, (b) Scene image with full dataset, and (c) Scene image with $4 \%$ of the data volume

This technique, named Spatial Filtering (SF), was first introduced in [23]. The wall scattering invariance together with the fact that wall reflections are relatively stronger than target reflections are the basis of another technique presented in [24], where it was shown that the wall subspace is usually captured in the most dominant singular values when applying singular value decomposition (SVD) to the measured data matrix. Both methods were extended to deal with compressive measurements in [16].

\section{PRoposed techniQue}

Our goal is to obtain estimates of $\alpha_{m}$ and $\Delta \tau_{n}$ such that the wall reflection can be modeled and, subsequently, subtracted from the total radar returns to render wall-free signals. The calculation of the Maximum Likelihood (ML) estimate of $\Delta \tau_{n}$ can be found by,

$$
\hat{\tau}_{n}=\arg \max \left|\mathbf{s}_{n}^{H} \breve{\mathbf{y}}_{n}\right|
$$

where $\breve{\mathbf{y}}_{n}=\varphi^{(n)} \mathbf{y}_{n}$ is the reduced set of frequencies measured at the $n$-th antenna location and with,

$$
\mathbf{s}_{n}=\left[\begin{array}{lll}
e^{-j \omega_{0}\left(\tau_{\mathrm{w}}-\tau_{n}\right)} & \cdots & e^{-j \omega_{M-1}\left(\tau_{\mathbb{w}}-\tau_{n}\right)}
\end{array}\right]^{T}
$$

for $\tau_{\mathrm{w}}-\Delta \tau \leq \tau_{n} \leq \tau_{\mathrm{w}}+\Delta \tau$. Note that we use prior knowledge of the wall standoff distance to obtain the value of $\tau_{\mathrm{w}}$. The time delay limits defined by $\Delta \tau$ should be selected appropriately depending on the roughness of the surface. Once the shift in time domain is estimated as $\Delta \hat{\tau}_{n}=\hat{\tau}_{n}-\tau_{\mathrm{w}}$, it can be used to obtain the estimate of the scale parameter $\alpha_{m}$ as,

$$
\hat{\alpha}_{m}=\frac{1}{Q_{\mathrm{n}}} \sum_{n=1}^{Q_{\mathrm{n}}} \frac{|\breve{y}(m, n)|}{\left|\hat{y}_{\mathrm{w}}(m, n)\right|}
$$

where $\hat{y}_{\mathrm{w}}(m, n)$ denotes the estimated wall contribution obtained from (4) using the estimated $\Delta \hat{\tau}_{n}$ and with $\alpha_{m}=1$. Using $\Delta \hat{\tau}_{n}$ and $\hat{\alpha}_{m}$, the wall reflections can be reconstructed for the reduced data volume and, thus, subtracted from the compressive measurements. The estimated wall reflection model for the reduced set of measurements is given by,

$$
\hat{y}_{\mathrm{w}}(m, n)=\hat{\alpha}_{m} \breve{y}_{\mathrm{w}, \mathrm{ref}}(m, n) e^{j \omega_{m} \Delta \hat{\tau}_{n}}
$$

\section{Simulation Results}

In this section, we evaluate the performance of the proposed technique using synthesized data. A stepped-frequency signal consisting of $M=728$ frequencies covering the $\omega_{0}=1$ to $\omega_{M-1}=3 \mathrm{GHz}$ frequency band was used for interrogating the scene. A monostatic synthetic aperture array, consisting of $N=67$ element locations with an inter-element spacing of $1.87 \mathrm{~cm}$ was employed. The array was located parallel to the front wall, and centered at $x=0 \mathrm{~m}$ in crossrange at a standoff distance of $z_{\text {off }}=1 \mathrm{~m}$. The roughness of the wall is simulated by adding a bias to the standoff distance. This bias is selected randomly in the range $0.5 \mathrm{~m}$ to $0.5 \mathrm{~m}$. The scaling factor $\alpha_{m} \sim \mathcal{N}\left(1,10^{-3}\right)$ is modeled as a Gaussian random variable. The scene consisted of a single point target located at $(0,1.59) \mathrm{m}$ (near the behind-the-wall area). The region to be imaged is chosen to be $5.61 \mathrm{~m}$ (down-range) $\times 4.88 \mathrm{~m}$ (crossrange) centered at $(0,3.59) \mathrm{m}$ and is divided into $N_{z}=73 \times$ $N_{x}=33$ pixels, respectively.

Figure 1(a) shows the image corresponding to the simulated scene obtained with conventional backprojection applied directly to the full raw dataset after perfect wall subtraction. We plot the image intensity with the maximum intensity value in the image normalized to $0 \mathrm{~dB}$. Fig. 1(b) shows the backprojection image obtained when the full dataset is not pre-processed. Finally, Fig. 1(c) depicts the backprojection image of the scene when using only $Q_{\mathrm{f}}=146$ uniformly selected frequencies and $Q_{\mathrm{n}}=14$ uniformly selected array locations, which represent $4 \%$ of the total data volume. For comparison, Fig. 1(b) and Fig. 1(c) have been normalized according to the maximum intensity value in both images. Clearly, data reduction translates into the appearance of clutter in the image, thereby challenging the detection of the targets behind the wall. If the spatial filtering technique summarized in Section III is applied using the reduced set of measurements, the image shown in Fig. 2(a) is obtained. It can be observed that the target is obscured due to the non-planar surface of the wall. If the proposed method is applied to the compressive measurements, the image shown in Fig. 2(b) is obtained. Now, the target can be easily identified. The proposed technique was applied to the same scenario but assuming specular wall reflections, i.e., with a complete flat wall. Fig. 2(c) shows the corresponding recovered image, where the same compressive 


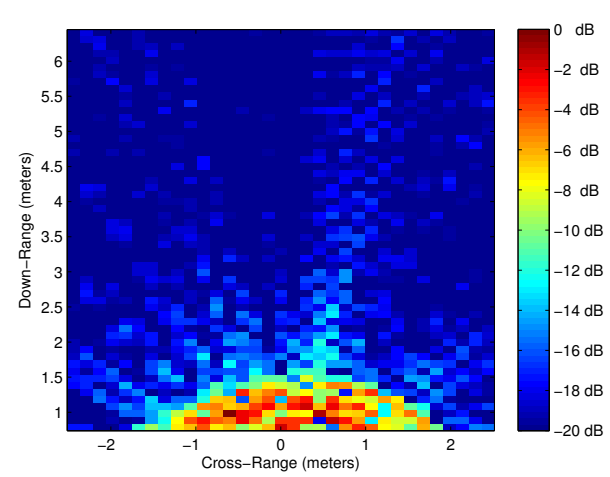

(a)

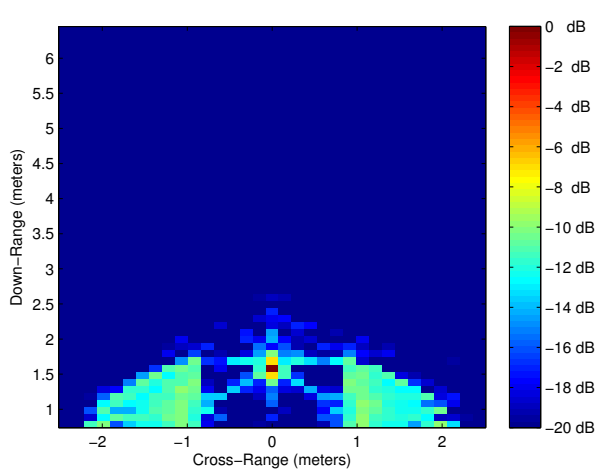

(b)

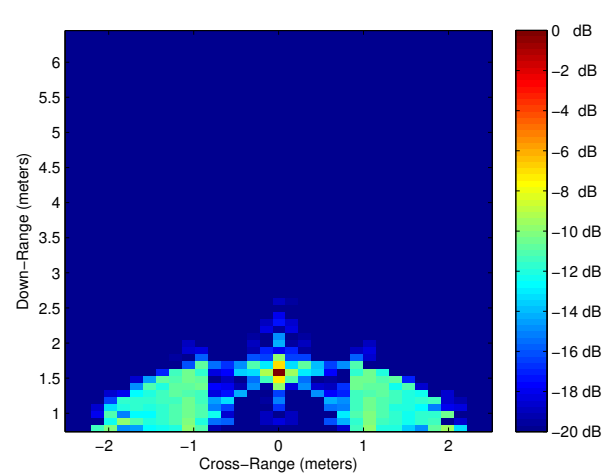

(c)

Fig. 2: Backprojected image with $4 \%$ of the data volume: (a) Spatial Filtering dealing with rough wall, (b) Proposed method dealing with rough wall, and (c) Proposed method dealing with flat wall.

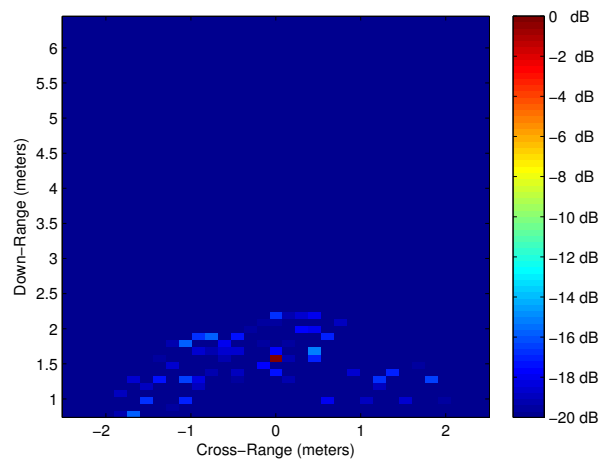

(a)

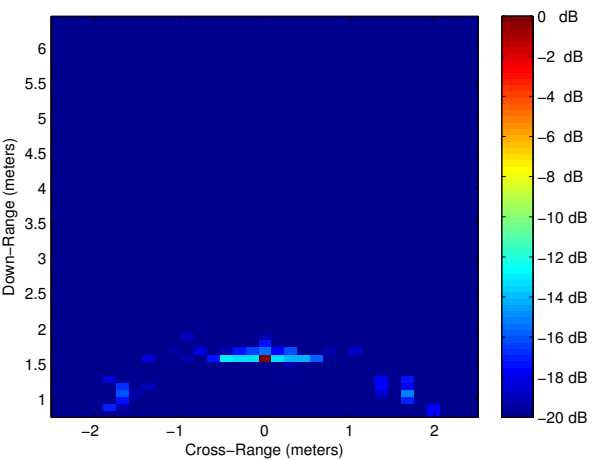

(b)

Fig. 3: $l_{1}$ reconstruction-based imaging results using the proposed technique with $4 \%$ of the data volume: (a) with rough wall, (b) with flat wall.

sampling pattern as in Fig. 2(b) has been considered. These results prove the efficiency of the proposed technique dealing with planar and non-planar walls.

Finally, Fig. 3(a) and Fig. 3(b) show the $l_{1}$ reconstruction based images corresponding to the data obtained after applying the proposed wall mitigation technique. It is evident that CS reconstructions provide an improvement over the corresponding backprojection results in terms of clutter reduction.

\section{CONCLUSION}

In this paper, we proposed a simple and effective technique to remove rough wall reflections from a random and small number of measurements. Essentially, the phase shift and the amplitude of the wall reflections are estimated from the compressive measurements using a MLE procedure. The estimated parameters are used to model EM wall returns, which are subsequently subtracted from the total radar returns, rendering wall-free signals. Simulation results demonstrated the effectiveness of the proposed technique when dealing with homogeneous and heterogeneous walls with significantly reduced data measurements

\section{ACKNOWLEDGMENT}

The work by M. G. Amin and F. Ahmad is supported by US Army Research Office/Army Research Lab under contract
W911NF-11-1-0536.

\section{REFERENCES}

[1] M. G. Amin (Ed.), "Through-the-Wall Radar Imaging," CRC Press, Boca Raton, FL, 2011.

[2] - "Special issue on Advances in Indoor Radar Imaging," J. Franklin Inst., vol. 345, no. 6, pp. 556-722, Sep, 2008.

[3] M. G. Amin and K. Sarabandi (Eds.), "Special issue on Remote Sensing of Building Interior," IEEE Trans. Geosci. Remote Sens., vol. 47, no. 5, pp. 1270-1420, May, 2009.

[4] E. Baranoski, "Through-Wall Imaging: Historical Perspective and Future Directions," J. Franklin Inst., vol. 345, no. 6, pp. 566-569, 2008.

[5] S. Borek, "An Overview of Through the Wall Surveillance for Homeland Security, Washington, DC, USA," Applied Imagery and Pattern Recognition Workshop, San, pp. 19-21, Dec, 2005.

[6] H. Burchett, "Advances in Through Wall Radar for Search, Rescue and Security Applications," Inst. of Eng. and Tech. Conf. Crime and Security, London, UK, pp. 511-525, Jun, 2006.

[7] P. Sevigny and D. DiFilippo, "A Multi-Look Fusion Approach to Through-Wall Radar Imaging," IEEE Radar Conference, Ottawa, Canada, Apr, 2013.

[8] E. Lavely, Y. Zhang, E. Hill III, Y.-S. Lai, P. Weichman, and A. Chapman, "Theoretical and experimental study of through-wall microwave tomography inverse problems," J. Franklin Inst., vol. 345, no. 6, pp. 592-617, 2008. 
[9] D. Donoho, "Compressed sensing," IEEE Trans. Inf. Theory, vol. 52, no. 4, pp. 1289-1306, Apr, 2006.

[10] E. J. Candes and M. B. Wakin, "An Introduction to Compressed Sampling," IEEE Signal Process. Mag., vol. 25, no. 2, pp. 21-30, March, 2008.

[11] Y. Yoon and M. G. Amin, "Compressed Sensing Technique for HighResolution Radar Imaging," Proc. SPIE, vol. 6968, pp. 69681A-169 681A-10, 2008.

[12] Q. Huang, L. Qu, B. Wu, and G. Fang, "UWB Through-Wall Imaging based on Compressive Sensing," IEEE Trans. Geosci. Remote Sens., vol. 48, no. 3, pp. 1408-1415, 2010.

[13] M. Amin, F. Ahmad, and W. Zhang, "Target RCS exploitations in compressive sensing for through wall imaging," 5th Int. Waveform Diversity and Design Conf., Niagara Falls, Canada, Aug., 2010.

[14] M. G. Amin (Ed.), "Compressive Sensing for Urban Radar," CRC Press, Boca Raton, FL, 2014.

[15] E. Lagunas, M. Amin, F. Ahmad, and M. Najar, "Wall Mitigation Techniques for Indoor Sensing within the Compressive Sensing Framework," IEEE Sensor Array and Multichannel Signal Processing Workshop, Hoboken, USA, Dec, 2011.

[16] _ _ "Joint Wall Mitigation and Compressive Sensing for Indoor Image Reconstruction," IEEE Trans. Geosci. Remote Sens., vol. 51, no. 2, pp. 891-906, Jan, 2013.

[17] _ - "Compressive Sensing for Through Wall Radar Imaging of
Stationary Scenes using Arbitrary Data Measurements," Int. Conf. on Information Science, Signal Processing and their Applications (ISSPA), Montreal, QC, Canada, Jul, 2012.

[18] M. Dehmollaian and K. Sarabandi, "Refocusing Through the Building Walls Using Synthetic Aperture Radar," IEEE Trans. Geosci. Remote Sens., vol. 46, no. 6, pp. 1589-1599, 2008.

[19] M. Tuncer and A. Gurbuz, "Ground Reflection Removal in Compressive Sensing Ground Penetrating Radars," IEEE Geosci. Remote Sens. Lett., vol. 9, no. 1, pp. 23-27, Jan, 2012.

[20] R. Millar, Maximum Likelihood Estimation and Inference, Statistics in Practice. John Wiley and Sons, Inc, New York, USA, 2011.

[21] M. G. Amin and F. Ahmad, "Wideband synthetic aperture beamforming for through-the-wall imaging," IEEE Signal Process. Mag., vol. 25, no. 4, pp. 110-113, Jul, 2008.

[22] J. A. Tropp and A. C. Gilbert, "Signal Recovery From Random Measurements Via Orthogonal Matching Pursuit," IEEE Trans. Inf. Theory, vol. 53, no. 12, pp. 4655-4666, Dec, 2007.

[23] Y. Yoon and M. G. Amin, "Spatial Filtering for Wall-Clutter Mitigation in Through-the-Wall Radar Imaging," IEEE Trans. Geosci. Remote Sens., vol. 47, no. 9, pp. 3192-3208, 2009.

[24] F. Tivive, M. Amin, and A. Bouzerdoum, "Wall Clutter Mitigation Based on Eigen-Analysis in Through-The-Wall Radar Imaging," Int Conf. Digital Signal Process., Corfu, Greece, pp. 1-8, Jul, 2011. 